\section{Corneo-conjunctival carcinoma in Uganda}

KM Waddell', RG Downing², SB Lucas³ and R Newton ${ }^{4}$

\begin{abstract}
Aims Since 1990, the incidence of conjunctival neoplasia has more than tripled in Uganda. It is known to be associated with exposure to solar ultraviolet radiation and to infection with the human immunodeficiency virus-1 (HIV). However, little is known about the most effective treatments. In this study, we report surgical outcomes among people with corneo-conjunctival squamous neoplasia in Uganda and investigate the role of HIV infection and other factors in the aetiology of the tumour.

Methods Country-wide enrolment of participants; removal and histology of suspect lesions; HIV counselling and testing; home visiting of participants to determine outcomes.
\end{abstract}

Results In 67 months between 1995 and 2001, 476 participants were enrolled ( 262 female, 214 male, median age 32 years). A total of 463 (97\%) had eye-conserving removal of the lesion and 13 had other surgery. For 414, the histology was squamous neoplasia (184 invasive carcinoma, 230 intraepithelial). The prevalence of HIV infection in cases was $64 \%$. In all, $96 \%$ were followed up for a median period of 32 months (range 0-81) after eyeconserving surgery during which time 13 (3.2\%) had a recurrence.

Conclusions Surgery resulted in a low recurrence rate during the follow-up period and had minimal complications. The prevalence of HIV among cases was higher than expected on the basis of data from the general population, although about a third of cases were HIV-negative and had normal CD4 counts. No new cofactors were identified.

Eye (2006) 20, 893-899. doi:10.1038/sj.eye.6702043; published online 3 February 2006

Keywords: conjunctiva; cancer; Uganda; HIV

\section{Introduction}

In the years before HIV, corneo-conjunctival intraepithelial neoplasia (CIN) and carcinoma (together called ocular surface squamous neoplasia (OSSN)) were reported to be more frequent in African countries including Uganda than in Europe and the USA. ${ }^{1-3}$ Recently using data from worldwide cancer registries it has been confirmed that incidence of OSSN increases markedly with proximity to the equator, presumably from increasing solar ultraviolet (UV) radiation. ${ }^{4}$ In the 1980 s there was a marked increase in the number of cases being seen in Uganda (KW, personal observation), particularly among younger people, suggesting a link with HIV infection. Studies in Rwanda, Uganda, and Malawi confirmed a roughly 10 -fold excess risk of the tumour in HIV-infected people compared to the uninfected, although the number of cases studied is so far only around $140 .^{5-8}$ Despite this association, about a third of cases are HIVseronegative, suggesting that the frequency has also risen among them. To date there have been only a few reports elsewhere of HIV-associated cases, mainly from the USA. ${ }^{9,10}$

OSSN is now an everyday clinical problem in many African countries, but management there has been little studied, and if mismanaged, the eye is lost and death can result. Surgery has always been the main treatment. ${ }^{11}$ The techniques described vary according to the surgeon's preference, but series are often small and follow-up incomplete, so that outcome is unclear, although recurrence seems common. Recently topical chemotherapy has been advocated, but guidelines are complex for resource-poor regions. ${ }^{12}$ Radiotherapy has sometimes been used, but is seldom available in Africa. This study therefore aimed to document a large series in Uganda treated uniformly with a surgical technique suitable for resource-poor areas, and to follow up the participants by home
${ }^{1}$ Ruharo Eye Hospital, Mbarara, Uganda

${ }^{2}$ CDC-Uganda, Uganda Virus Research Institute, Entebbe, Uganda

${ }^{3}$ Guy's, King's and St Thomas' School of Medicine, London, UK

${ }^{4}$ Epidemiology and Genetics Unit, Department of Health Sciences, University of York UK

Correspondence: KM Waddell, PO Box 4008, Kampala, Uganda Tel: + 25641 200222; Fax: + 25641200486. E-mail: aim-uganda@ aimint.net

Received: 23 February 2005 Accepted in revised form: 24 March 2005 Published online: 3 February 2006 
visits to document outcome. It also aimed to investigate the aetiology further.

\section{Materials and methods}

\section{Participants}

From November 1995 to May 2001 in country-wide clinics, anyone with a suspect corneo-conjunctival lesion was offered removal and histology, and enrolment in a follow-up study with home visits. HIV serology was also offered after pretest counselling. Lesions were photographed and details of the eyes and general health were recorded and analysed in EPI INFO version 6 .

\section{Consent and ethical approval}

Information about the disease, its treatment, and HIV testing was given in private in vernacular by counsellors, and consent confirmed by signature or thumbprint. The study was approved by the Science and Ethics Committee of the Uganda Virus Research Institute, and by the Uganda National Council for Science and Technology.

\section{Surgery}

Lesions judged removable leaving a normal eye were dealt with as described below. If this was impossible, enucleation was advised. If too advanced for this, incision biopsy was performed to confirm the diagnosis, and referral offered for further management. Surgery was carried out by one surgeon (KW) using binocular magnifying loupes.

\section{Anaesthesia}

Subjects were anaesthetised with amethocaine drops, and retrobulbar, subconjunctival, and facial nerve block with $2 \%$ lignocaine.

\section{Lesions confined to conjunctiva}

The resection started $3 \mathrm{~mm}$ beyond the visible tumour edge, or at the corneal margin if less. On the deep surface resection was in one block to bare sclera or muscle. Lamellar sclerectomy was included only if fixation to the globe was suspected. The sclera was lightly cauterised especially at the corneal edge to destroy any fragments.

Lesions involving cornea

If superficial to Bowman's layer, it was peeled off by semi-sharp dissection. If fixed, shallow keratectomy was performed. The area was wiped with ethanol to destroy any fragments.

\section{Closure of the conjunctiva}

It was carried out either by pedicle flap graft of superior bulbar conjunctiva or by mobilising adjacent conjunctiva.

\section{Aftercare}

Topical antibiotic was instilled and a dressing applied till next day. Then the patient put in antibiotics for 1 or 2 weeks. If hyperaemia remained excessive, topical steroid drops were used for a further 1 or 2 weeks until healing was complete.

\section{Serology and histopathology}

Venous blood was taken and screening tests for HIV antibodies were performed with confirmation at the Uganda Virus Research Institute (two enzyme immunoassay tests in parallel, with Western blot if required). T-lymphocyte subset counts were performed using FACS Count (Becton Dickinson). HIV subtyping was performed by PCR EIA (Roche Diagnostics) using in-house subtype-specific primers and probes. Biopsies went to St Thomas' Hospital, London, for histopathology. CIN was classified into three stages according to one-, two- or three-thirds thickness being dysplastic. Pieces of larger tumours were frozen in liquid nitrogen for future viral and host genomic studies. Some cases were investigated for the presence of antibodies against HIV in tears using Western Blot.

\section{Follow-up}

Participants returned to the clinic if feasible to monitor healing and receive HIV results with post-test counselling. For long-term follow-up, field workers traced them to homes or workplaces, where the surgeon visited them. Visual acuity was recorded and eyes examined with binocular loupes. If recurrence occurred, re-operation was arranged. Intraocular pressure was measured 3 months or more after surgery by Perkins applanation tonometry. General health was noted, with referral to other services if needed. Follow-up information was divided into two categories: 'direct' (from the surgeon's examination or the participant's communication) and 'reported' (from wider sources, usually after death). Follow-up ended in May 2003 after $7 \frac{1}{2}$ years.

\section{Results}

Participants, surgery, and histopathology

Figure 1 shows the flow of participants through the study. Their median age was 32 years, (interquartile range (IQR) 28-40; range 14-77). The side of the lesion 
was right for $251(53 \%)$ and left for $225(47 \%)$ and the cornea was involved in $47 \%$. In all, 19 were already recurrences at enrolment. Sclerectomy was included in 73 (16\%) (of these, $69 \%$ proved to be invasive), and keratectomy in 107 (23\%) (of these, 53\% proved invasive). Figure 2 shows the positions of the estimated centres of the first 352 lesions in the series. Tables 1 and 2 show the histology of the initial lesion for all 476 participants stratified by sex and HIV status. All the neoplastic lesions were squamous except one muco-epidermoid carcinoma. Fifteen also had removal of second primary tumours (12 in the opposite eye and three in opposite quadrant of the

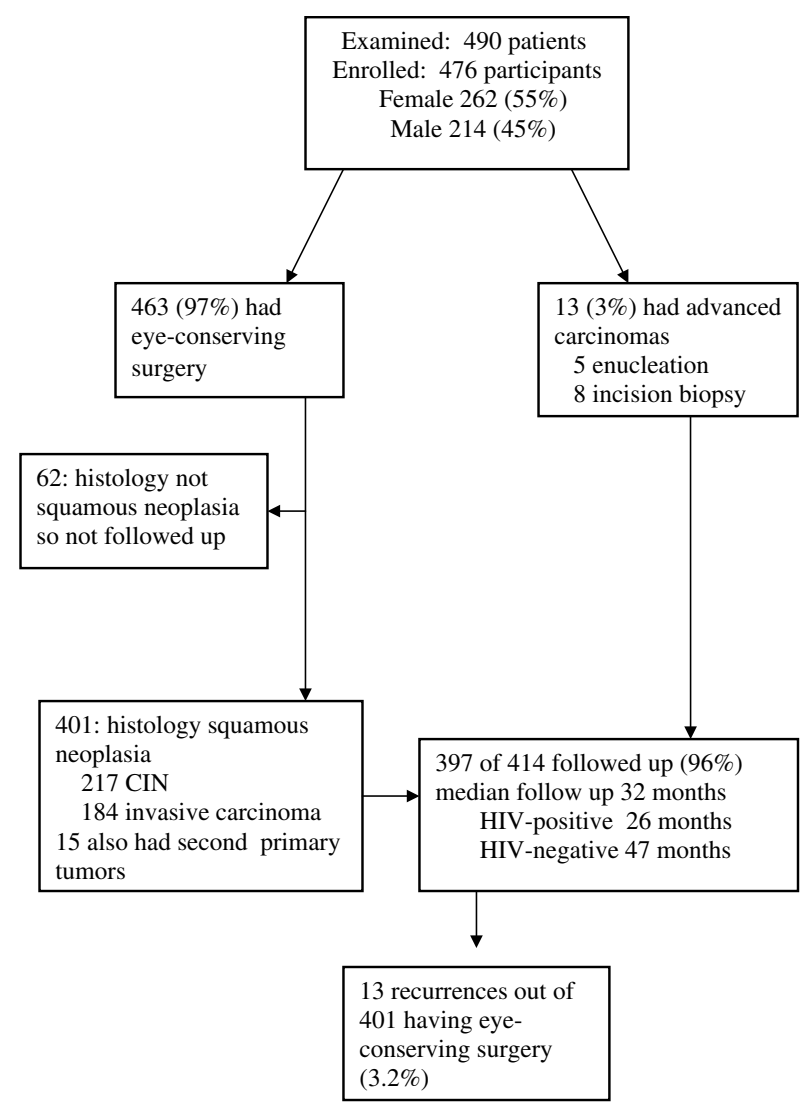

Figure 1 Flow diagram of participants through the study.
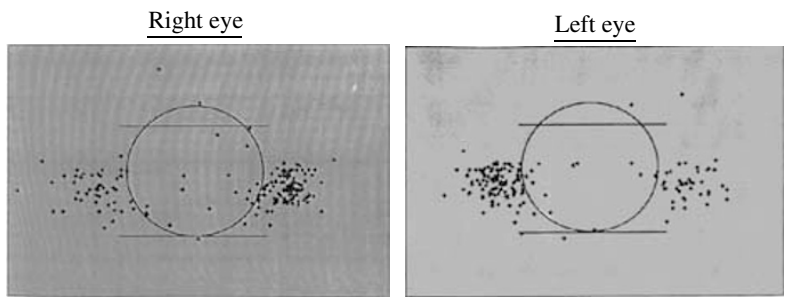

Figure 2 Estimated centres of 352 tumours relative to the cornea and eyelids for right and left eyes showing almost all are in the interpalpebral area. same eye) of which 12 were various stages of CIN and three were invasive carcinoma.

\section{HIV status, T-lymphocyte subset counts, and HIV subtype}

At enrolment HIV serology was obtained for 448 (94\%) participants; $64 \%$ of those with OSSN $(n=386)$ and $31 \%$ of those without it $(n=62)$ were positive (Tables 2 and 3$)$. According to the WHO Clinical Staging System, $76 \%$ of the 248 HIV-positive participants with OSSN were in stage 1 (asymptomatic), $13 \%$ were in stage 2 , and $10 \%$ in stage $3 \mathrm{HIV}$ disease. ${ }^{13}$ Two $(1 \%)$ were in stage 4 (AIDSdefining), one of them also having conjunctival Kaposi's sarcoma and another having early tuberculous meningitis. Table 2 shows histology against HIV status. The median age among cases, if HIV positive $(n=248)$, was 33 years (IQR 28-40; range 14-65), and if HIV negative ( $n=138), 32$ years (IQR 26-45; range 17-77). All but one of the 15 with second primary tumours were HIV positive. Table 3 shows CD $4+$ T-lymphocyte counts and the CD4 + /CD8 + ratios at enrolment for 159 participants subdivided by serostatus. Sub-typing of HIV was performed on blood from 90 seropositive participants. Two subtypes (A and D) of HIV-1 group M were identified in about equal proportions, and a few had both subtypes (either coinfection or recombinant virus). No sequences of HIV-1 group O or of HIV-2 were identified. No HIV sequences were identified from any of 136 participants who were seronegative for HIV-1 and HIV-2. Tears from 20 participants (six HIV-seropositive

Table 1 Histology subdivided by gender (number (\%))

\begin{tabular}{lrcr}
\hline Histology & Female & Male & \multicolumn{1}{c}{ Total } \\
\hline Not CIN/carcinoma & $31(12)$ & $31(15)$ & $62(13)$ \\
CIN 1 & $33(13)$ & $25(12)$ & $58(12)$ \\
CIN 2 & $35(13)$ & $20(9)$ & $55(12)$ \\
CIN 3 & $59(23)$ & $58(27)$ & $117(25)$ \\
Invasive carcinoma & $104(39)$ & $80(37)$ & $184(38)$ \\
Total & & & \\
\hline
\end{tabular}

Table 2 Histology and HIV serostatus (number (\%))

\begin{tabular}{lrcr}
\hline Histology & HIV-positive & HIV-negative & \multicolumn{1}{c}{ Total } \\
\hline Not CIN or carcinoma & $19(31)$ & $43(69)$ & $62(100)$ \\
CIN 1 & $32(60)$ & $21(40)$ & $53(100)$ \\
CIN 2 and 3 & $105(65)$ & $57(35)$ & $162(100)$ \\
Invasive carcinoma & $111(65)$ & $60(35)$ & $171(100)$ \\
Total CIN/carcinoma & $248(64)$ & $138(36)$ & $386(100)$
\end{tabular}


Table $3 \mathrm{CD} 4+$ T-lymphocyte subset counts and CD4+/ $\mathrm{CD} 8+$ ratios by HIV status (median cells $/ \mu \mathrm{l}$ (IQR); ratio (IQR))

\begin{tabular}{lcc}
\hline Histology & $\begin{array}{c}\text { HIV-positive } \\
\mathrm{N}=112\end{array}$ & $\begin{array}{c}\text { HIV-negative } \\
\mathrm{N}=47\end{array}$ \\
\hline Not CIN/carcinoma & $230.5(88-550)$ & $820.5(572-1050)$ \\
CIN 1 & $104(59-263)$ & $890(697-933)$ \\
CIN 2 and CIN 3 & $115.5(56-219)$ & $752(685-858)$ \\
Invasive carcinoma & $111(63-218)$ & $750(660-842)$ \\
All CIN/carcinoma & $111(62-221)$ & $763(675-890)$ \\
CD4/CD8 ratio & $0.13(0.07-0.22)$ & $1.22(0.97-1.75)$ \\
\hline
\end{tabular}

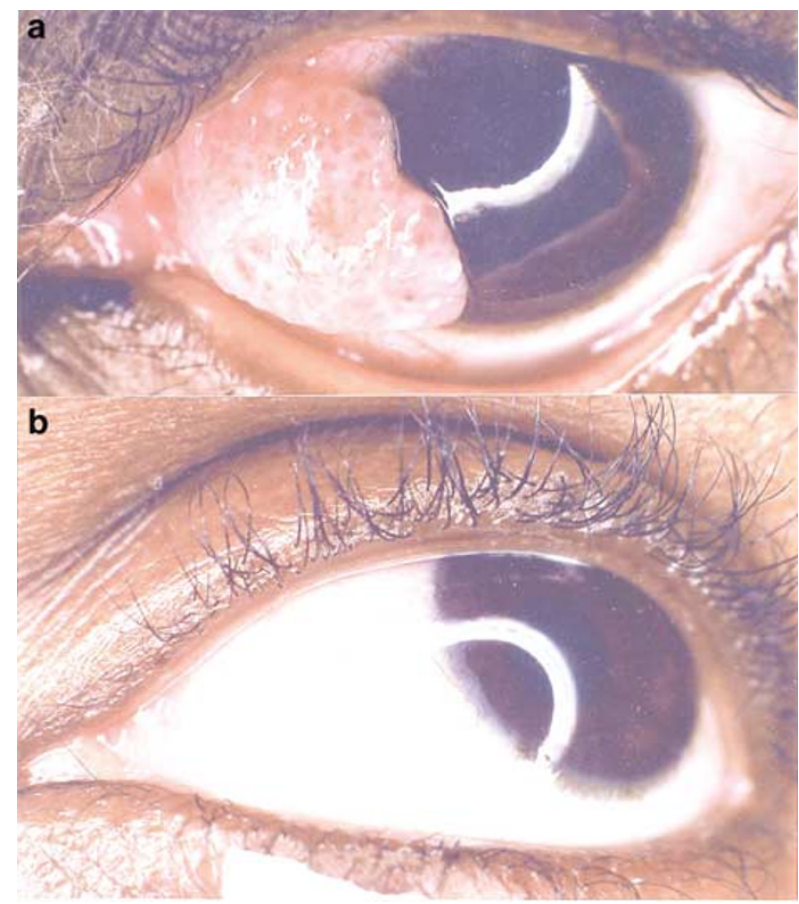

Figure 3 (a) Invasive conjunctival squamous cell carcinoma (left eye of a 28-year-old HIV-positive female). (b) After removal; there was no recurrence at 72 months.

and 14 negative) were tested for antibodies to HIV by Western blot analysis; all were concordant with blood results.

\section{Outcome}

Follow-up

Figure 1 shows the numbers followed up and Figure 3 shows a conjunctival lesion before and after treatment. For the 414 with CIN or invasive carcinoma, the median follow-up time was 32 months (IQR 16-48). Of these, if seropositive $(n=248)$, it was 26 months (IQR 10.5-41.5), and if negative $(n=138), 47$ months (IQR 36-59). For all invasive cases regardless of HIV serostatus $(n=184)$ it was 29 months (IQR 13-46). The ranges of all were from 0 to 81 months. Of the total months of follow-up, $88 \%$ was by direct information and $12 \%$ reported.

\section{Recurrence}

Recurrences after eye-conserving tumour removal were found in 13 (six from CIN and seven from invasive carcinoma). Ten were small and were treated by repeat surgery; none had recurred again by the end of the study or death. Of the three others, one had enucleation but died from HIV-related causes, the second had multiple repeat removals, and the third had exenteration and radiotherapy; the last two were well at latest follow-up. The repeat operations were performed at median of 13 months (range 4-36). In addition, five others developed corneal 'frosting', which was possibly early dysplasia but was too fragile for histology: two cleared with topical 5 -fluorouracil and the others did not progress. Of the 13 participants not having eye-conserving surgery because of advanced tumour, nine are known to have died; three others with massive tumour are presumed dead. Of the five who underwent enucleation as the initial operation, three died with orbital recurrence, one died from HIV-related illness without recurrence, and one remains well at 15 months. No distant metastases were found in any case, not even to local lymph nodes.

\section{HIV-associated mortality}

Taking the 387 cases with CIN or carcinoma and known HIV serology, death was recorded for 163 of 249 (65\%) seropositive cases at a median of 20 months, and 10 of $138(7 \%)$ seronegative cases at a median of 20.5 months.

\section{Visual acuity outcome}

Table 4 gives acuity categories for the eye with the lesion for all 476 participants before surgery and for 316 after. The main reason for preoperative loss was tumour obscuring the visual axis or invading, and for

Table 4 Categories of visual acuity of the operated eyes (number (\%))

\begin{tabular}{lccc}
\hline Category & $\begin{array}{c}\text { Snellen } \\
\text { acuity }\end{array}$ & $\begin{array}{c}\text { Before } \\
\text { operation }\end{array}$ & $\begin{array}{c}\text { After } \\
\text { operation }\end{array}$ \\
\hline Normal & $6 / 5-6 / 18$ & $425(89)$ & $294(93)$ \\
Visual impairment & $6 / 24-6 / 60$ & $20(4)$ & $8(3)$ \\
$\begin{array}{l}\text { Severe visual } \\
\text { impairment }\end{array}$ & $5 / 60-3 / 60$ & $4(1)$ & $2(1)$ \\
Blind & $\begin{array}{l}2 / 60-\text { light } \\
\text { perception }\end{array}$ & $18(4)$ & $3(1)$ \\
No light perception & & $9(2)$ & $9(3)$ \\
Total & & $476(100)$ & $316(100)$ \\
\hline
\end{tabular}


postoperative improvement was its removal. Nine participants with initially good vision experienced major visual loss as follows. Two developed cataract but regained normal vision after extraction and lens implantation; both were HIV-negative. Two had uveitis (bilateral and preceding surgery in one, the other in the operated eye following surgery); both died of HIVrelated causes. One (HIV-positive) had 12 excisions of carcinoma ending with corneal scarring. Two developed retinopathy; one was an infectious retinitis presumed due to cytomegalovirus (CD4 + count 60 cells $/ \mu \mathrm{l})$. The other developed a pigmentary retinopathy with loss of visual field but normal acuity; both died of HIV-related causes. Two (both HIV-positive) had invasion of the globe by tumour following local removal; one had enucleation and the other exenteration.

\section{Postoperative intraocular pressure}

This was measured in 204 participants. The mean was $14.4 \mathrm{mmHg}$ for the operated eye (range 4-27) and $14.6 \mathrm{mmHg}$ in the fellow eye (range 5-26). Four were above $21 \mathrm{~mm}$; three of these had bilateral ocular hypertension, and one was raised only in the operated eye; all were HIV-negative. Of the participants, 11 had pressures below $10 \mathrm{~mm}$; all were HIV-positive and no pathology was found to account for the low pressures.

\section{Discussion}

\section{Main findings of the study}

1. There is a strong association between OSSN and HIV-1 infection applying to all tumour stages and probably accounting for the marked increase in incidence noted in Uganda since the advent of the HIV epidemic. The HIV-1 subtypes that are the common ones in Uganda (A and D) and no outlier strains or HIV-2 were found. About a third of participants were HIV-negative.

2. Surgery by the technique described (applicable to resource-poor areas) resulted in a low recurrence rate $(3.2 \%)$ on medium term follow-up, and leaves a normal eye in most cases. Late presentation can result in death with massive orbital involvement. The few who lost vision did so from tumour or HIV-related causes, not from surgery.

3. HIV-positive participants were markedly immunosuppressed at the time of diagnosis (although they mostly had only minor symptoms) and their early mortality was high. Those seronegative were not found to be immunosuppressed.

4. The site of almost all tumours in the interpalpebral area of the conjunctiva supports the concept that UV radiation is a cofactor in the aetiology.

\section{Diagnosis and treatment}

The large number enrolled in a short period reflects the marked recent rise in frequency. The study shows the need to remove any suspicious lesion, because even those as small as $2 \mathrm{~mm}$ can be invasive carcinomas. Endophytic carcinomas can be especially deceptive, sometimes appearing like thick pingueculae. The surgical technique used differed in some details from others published. It excised a narrower rim $(3 \mathrm{~mm})$ of visibly normal conjunctiva than some recommend, but histology confirmed that almost always the visible margin of the tumour corresponds to the limit of neoplastic tissue. Diffuse lesions were rare and were visible as such, and so $3 \mathrm{~mm}$ is adequate. Sclerokeratectomy was used only when deep invasion was suspected, and many of these proved noninvasive or inflammatory, so assessment of fixation can be deceptive, especially close to the cornea where Tenon's capsule is deficient. Cryotherapy was not used, being unavailable in most locations, but also because it is not yet evidence based and complications are recorded.

\section{Outcome}

\section{Recurrence}

In this study the documented rate is low compared with some others (3.2\% after eye-conserving surgery), but this must be interpreted with reference to the length of follow-up. The median time is relatively short (32 months), though for seronegative cases it is 47 months and the range goes to 81 months. Since so many participants died early, the median cannot now rise much; however, long follow-up continues. On the other hand, the follow-up was unusually complete for a nation-wide study in Africa (information on $96 \%$ of participants). Over 1500 postoperative examinations were made to spread over $7 \frac{1}{2}$ years. These were mostly in homes not clinics, but conjunctival lesions are easily visible and can be detected with magnifying loupes even when minute. Unexpectedly about half the recurrences were from intra-epithelial lesions; all were HIV-positive. Late recurrence of CIN after several years has been reported from Australia (though their early recurrence rate was also high). ${ }^{14}$ Recurrence is presumably mostly from incomplete removal, but it may be from second primary lesions at the same site, as the study shows these can occur at other sites. With invasive carcinoma, histology showed that the plane of deep resection sometimes transected the tumour (as expected from the narrow space between conjunctiva and sclera), yet most of these did not recur during the study. This probably points to the importance of cautery and alcohol after resection to destroy any remaining tumour fragments. 
Mohs' micrographic surgery has been recommended, with immediate histology and possible further surgery next day, but this would be impractical in much of Africa. ${ }^{15}$ Topical anti-tumour chemotherapy is now being recommended, but this requires cytology or incision biopsy for diagnosis followed by prolonged observation, so is again difficult in Africa. ${ }^{12}$ Thus, surgery by excision biopsy is likely to remain the standard in Africa, especially if this low recurrence rate is maintained.

\section{Visual outcome}

The outcome in terms of visual acuity was satisfactory. Although data on postoperative acuity were incomplete, Table 4 shows overall an improvement in categories of vision, mostly due to removal of tumour. The seven cases with permanent major loss were all accountable by the tumour or by complications of HIV, not the surgery. IOP was measured in a sample after 3 months in case dissection at the limbus had affected it, but there was no difference in mean pressures between operated and unoperated eyes, with only one raised pressure after operation (and three with bilateral ocular hypertension). Seropositive cases can have unexplained low pressures.

\section{Mortality}

As expected from their low CD4 + counts, more than half the HIV-infected participants died during the study, presumably mostly from HIV-related causes. Highly active antiretroviral therapy (HAART) only became available after the start of the study, and was financially prohibitive until the final months. It has been stated that there are very few recorded deaths from the tumour itself, but this study shows that late presentation or reluctance to accept surgery can result in death with massive tumour. ${ }^{1}$ On the other hand, it was encouraging that most participants arrived in the curable stages.

\section{HIV and immunosuppression}

The prevalence of HIV infection among cases (64.3\%) was similar to that reported in other studies. ${ }^{6,7}$ Obtaining suitable controls for calculating odds ratios relating to the impact of HIV infection on tumour risk was problematic. Participants whose histology proved not to be OSSN were initially chosen but were few. Also these controls had a higher HIV-seropositivity rate than expected $(30.6 \%)$ on the basis of population rates. It was, therefore, decided not to estimate odds ratios. Even so the association cannot be doubted, and it applies to all tumour stages (Table 2). The subtypes of HIV-1 found were the common ones in Uganda (A and D).

Compartmentalisation of infection to the conjunctival sac (as can occur with the urogenital tract) did not occur in a small sample. Therefore, outlier strains or localised HIV infection cannot explain the large number of seronegative cases. The CD4 + T-lymphocyte counts showed that HIV-infected cases were much more immunosuppressed than suspected from their initial good health (Table 3). Their median count at enrolment was 111 cells $/ \mu 1$. In another study in Uganda the median count at diagnosis of an AIDS-defining disease was 150 cells $/ \mu$ l. ${ }^{16}$ By the WHO classification, only two were stage 4 (AIDSdefining), but if another classification is used in which a CD4 + count below 200 is AIDS-defining, the majority are included. ${ }^{17}$ Kaposi's sarcoma can regress strikingly with HAART, but it remains to be seen how a carcinoma would react.

\section{Frequency of the tumour}

A series from the Mayo Clinic in the USA took 64 years to accrue 120 cases, and only 19 were initially invasive. ${ }^{18}$ Although the frequency in Africa was suspected to be higher than in Europe and the USA even before HIV, 2,3 the enrolment in this study of over 400 cases in $5 \frac{1}{2}$ years points to a large recent increase in incidence.

Surprisingly, about a third are not HIV infected and are not clearly immunosuppressed from other causes (as measured by CD4 count), yet they are so many (137 in this study) that they have very likely also markedly increased. A study from the cancer registry in Kampala, Uganda, confirms the increase; analysis of time trends in eye cancer showed a four-fold increase between the 1960s and 1990s, although the HIV status of cases was unknown. ${ }^{19}$ It is suggested that when immunosuppression is involved in tumorigenesis, an oncogenic virus is usually implicated even in those not immunosuppressed. ${ }^{20}$ This points to HIV acting not directly but by facilitating another agent whose transmission has been boosted in the community as well as in HIV-infected individuals (as occurs with Kaposi's sarcoma). A search is ongoing for candidate cofactor viruses for the carcinoma, including strains of human papillomavirus. ${ }^{21,22}$

\section{Other cofactors}

This study supports the likelihood that UV irradiation plays a part, at least in determining the site of the tumour, because Figure 2 shows that almost all tumours are in the exposed interpalpebral area. Also they predominate in the nasal quadrant like pterygia, which are also related to UV radiation. The conjunctiva is the one site in African people not protected from the sun by heavy pigmentation, and a role for UV is plausible by analogy with skin cancer. Of other suggested cofactors, none was supported by the study. Very few smoked tobacco, and if smoke itself was a cofactor, older women cooking over open fires would be expected to be affected, but this is not so. None showed clinical signs of vitamin 
A deficiency, nor of active trachoma (although one tumour was in an eye blind from Trachomatous trichiasis). The role of other cofactors in OSSN remains poorly understood and it is not yet known if the incidence will rise outside of Africa as the HIV epidemic spreads.

\section{Acknowledgements}

We thank Christoffel Blindenmission for financing the work, Mr J Magyezi and Mr D Tukwasibwe for helping in the care of the participants, and Ms D Casabonne for help with statistical analysis.

\section{References}

1 Lee G, Hirst L. Ocular surface squamous neoplasia. Surv Opthalmol 1995; 39: 429-450.

2 Clear A, Chirambo M, Hutt M. Solar keratosis, pterygium, and squamous cell carcinoma of the conjunctiva in Malawi. Br J Ophthalmol 1979; 63: 102-109.

3 Templeton A. Tumours of eye and adnexa. Recent Results Cancer Res. 1973; 43: 203-214.

4 Newton R, Ferlay J, Reeves G, Beral V, Parkin D. Effect of ambient solar ultraviolet radiation on incidence of squamous-cell carcinoma of the eye. Lancet 1996; 347: 1450-1451.

5 Kestelyn P, Stevens A, Ndayambaje A, Hanssens M, van de Perre P. HIV and conjunctival malignancies. Lancet 1990; 336: 51-52 (letter).

6 Ateenyi-Agaba C. Conjunctival squamous-cell carcinoma associated with HIV infection in Kampala, Uganda. Lancet 1995; 345: 695-696.

7 Waddell K, Lewallen S, Lucas S, Atenyi-Agaba C, Herrington C, Liomba G. Carcinoma of the conjunctiva and HIV infection in Uganda and Malawi. Br J Ophthalmol 1996; 80: 503-538.

8 Newton R, Ziegler J, Beral V, Mbidde E, Carpenter L, Wabinga $\mathrm{H}$ et al. The Uganda Kaposi's Sarcoma Study Group. A case control study of human immunodeficiency virus infection and cancer in adults and children residing in Kampala, Uganda. Int J Cancer 2001; 92: 622-627.
9 Kim R, Seiff S, Howes E, O'Donnell J. Necrotizing scleritis secondary to conjunctival squamous cell carcinoma in acquired immunodeficiency syndrome. Am J Ophthalmol 1990; 109: 231-233.

10 Winward K, Curtin V. Conjunctival squamous cell carcinoma in a patient with human immunodeficiency virus infection. Am J Ophthalmol 1989; 107: 554-555.

11 Shields J, Shields C, De Potter P. Surgical management of conjunctival tumors. Arch Opthalmol 1997; 115: 808-815.

12 Basti S, Macsai M. Ocular surface squamous neoplasia: a review. Cornea 2003; 22: 687-704.

13 World Health Organization. Proposed clinical staging system for HIV infection and disease. Wkly Epidemiol Rec 1990; 65: 222

14 Tabin G, Levin S, Snibson G, Loughnan M, Taylor H. Late recurrence and the necessity for long-term follow-up in corneal and conjunctival intraepithelial neoplasia. Ophthalmology 1997; 104: 485-492.

15 Buuns D, Tse D, Folberg R. Microscopically controlled excision of conjunctival squamous cell carcinoma. Am J Ophthalmol 1994; 117: 97-102.

16 Morgan D, Malamba S, Orem J, Mayanja B, Okongo M, Whitworth J. Survival by AIDS defining condition in rural Uganda. Sex Transm Inf 2000; 76: 193-197.

17 Centers for Disease Control. 1993 revised classification system for HIV infection and expanded surveillance case definition of AIDS among adolescents and adults. MMWR 1993; 41: 1-19.

18 Erie J, Campbell R, Liesegang T. Conjunctival and corneal intraepithelial and invasive neoplasia. Ophthalmology 1986; 93: 176-183.

19 Wabinga H, Parkin D, Wabwire-Mangen F, Nambooze S Trends in cancer incidence in Kyadondo county, Uganda, 1960-1997. Br J Cancer 2000; 82: 1585-1592.

20 Beral V, Newton R. Overview of the epidemiology of immunodeficiency-associated cancers. J Natl Cancer Inst Monogr 1998; 23: 1-6.

21 Newton R, Ziegler J, Ateenyi-Agaba C, Bousarghin L, Casabonne D, Beral V et al. The Uganda Kaposi's Sarcoma Study Group. The epidemiology of conjunctival squamous cell carcinoma in Uganda. Br J Cancer 2002; 87: 301-308.

22 Waddell K, Magyezi J, Bousarghin L, Coursaget P, Lucas S, Downing $\mathrm{R}$ et al. Antibodies against human papillomavirus type 16 (HPV-16) and conjunctival squamous cell neoplasia in Uganda. Br J Cancer 2003; 88: 2002-2003 (letter). 